\title{
Focused Ultrasound Lipolysis in the Treatment of Abdominal Cellulite: An Open-Label Study
}

\author{
Hamideh Moravvej ${ }^{1,2 *}$, Zahra Akbari' ${ }^{1}$, Shahrzad Mohammadian', Zahra Razzaghi' \\ ${ }^{1}$ Laser Application in Medical Sciences Research Center, Shahid Beheshti University of Medical Sciences, \\ Tehran, Iran \\ ${ }^{2}$ Skin Research Center, Shahid Beheshti University of Medical Sciences, Tehran, Iran
}

\author{
Correspondence to \\ Hamideh Moravvej, MD; \\ Skin Research Center, Shahid \\ Beheshti University of Med- \\ ical Sciences, Tehran, Iran. \\ Tel: +98-2122741507; Fax: \\ +982122744393; Email: \\ hamideh_moravvej@yahoo.com
}

Published online 28 June 2015

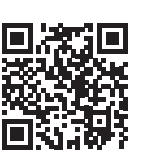

\begin{abstract}
Introduction: Despite a growing popularity of noninvasive ultrasonic lipolysis procedure, there is a lack of evidence about the efficacy of this method. This study was performed to evaluate the efficacy of focused ultrasonic lipolysis on abdominal cellulite treatment.

Methods: Twenty-eight consecutive subjects (age: $37.8 \pm 8$ years) underwent weekly transdermal focused ultrasonic lipolysis (Med Contour, General Project Ltd., Florence, Italy) and vacuum drainage for a maximum of eight sessions. Largest abdominal girth and 2 lines at $4 \mathrm{~cm}$ to $7 \mathrm{~cm}$ distance above and under it were located as fixed points of measurements. The mean value of the three fixed lines was considered as the abdominal circumference. Subjects were evaluated using measurements of circumference, immediately after and 3 weeks after the final treatment and compared using paired $t$ test.

Results: One hundred ninety-four ultrasonic lipolysis procedures were performed on 28 subjects. A statistically significant $(P<.001)$ average of $1.89 \mathrm{~cm}(95 \% \mathrm{Cl}: 1.63-2.02 \mathrm{~cm})$ decrease of circumference value was observed in each session of ultrasonic lipolysis. The mean pretreatment to posttreatment circumference reduction was $8.21 \mathrm{~cm}(95 \% \mathrm{Cl}$ : 6.38-10.04, $P<.001)$ that declined to $7 \mathrm{~cm}(95 \% \mathrm{Cl}: 3.2-10.8, P<.001)$ at the 3 -month follow-up visit.

Conclusion: Focused ultrasonic lipolysis appears to be an effective method for reduction of abdominal cellulite, although some amount of circumference reduction reversal may be observed in long term follow-up visit.

Keywords: Cellulite; Abdominal circumference; Lipolysis
\end{abstract}

\section{Introduction}

Despite acceptable efficacy and safety of conventional liposuction, postoperative complications such as seroma collection, fibrosis, pain, a few death reports and a prolonged recovery time are drawbacks of this treatment modality which are related to the invasiveness of this technique. ${ }^{1-4}$ Moreover, the second session of liposuction, especially over the subcutaneous fibrous area is less successful, which results in improper tightening of skin. ${ }^{5}$ Recently, less invasive methods of body contouring have been proposed that have been used alternatively or concomitantly with conventional tumescent liposuction. ${ }^{6}$ New researches indicate that ultrasonic waves can make structural alteration in subcutaneous fat deposition without negative effect on skin. Reduction of the intercellular pressure in fixed temperatures results in the development of millions of micron bubbles which leads to dramatic explosion of the latter due to repeated cycles of enlargement and shrinkage. This process generates a lot of energy that disrupts adipose cell wall and releases its lipid content which is finally transported to the hepatobiliary system via lymphatic drainage. This system, which is labeled "Cavitation," is hypothesized to result in a decrease of the local adipose tissue volume. Accordingly, a number of noninvasive lipolysis systems based on ultrasonic waves have been introduced for reduction of subcutaneous cellulite. ${ }^{1,7-10}$

The growing popularity of noninvasive ultrasonic lipolysis for cellulite reduction has given rise to concerns about the effectiveness of this procedure. To our knowledge, limited studies have been focused on clinical outcomes of focused ultrasonic lipolysis and there is controversy over the long-lasting effects of this procedure. ${ }^{9-12}$ This study was performed to assess the short-term as well as long-term effects of focused ultrasonic lipolysis on abdominal contouring.

\section{Methods \\ Patients \\ Twenty-eight consecutive subjects (27 females, 1 male; age: $37.8 \pm 8$ years) with local abdominal obesity referred to cosmetic clinic of Shohada-e Tajrish hospital for body}


contouring were recruited in the study. According to National Cholesterol Education Program's Adult Panel III (2005), abdominal obesity is defined as an abdominal circumference $\geq 102 \mathrm{~cm}$ for men and $\geq 88 \mathrm{~cm}$ for women. The patients with history of liver or renal failure, autoimmune disease, malignancy, pregnancy or lactation, local metal prosthesis, pace maker and anticoagulating medication were excluded. After full disclosure of the necessary information, all subjects signed an informed consent. The study protocol was approved by the Institutional Review Board on Human Research and conformed to the guideline of the 1975 Declaration of Helsinki.

\section{Ultrasonic Lipolysis}

Each patient underwent focused ultrasonic lipolysis (cavitation) of the abdominal area and vacuum drainage weekly for a maximum of 8 sessions. No preoperative preparation such as local anesthesia, sedation or prophylactic antibiotic was administered. The patients were informed to avoid dietary changes during the treatment process in order to eliminate the effect of weight loss on final results. The external ultrasonic lipolysis was performed using Med Contour system (General Project Ltd., Florence, Italy) that contains 2 manual hand pieces. The lipolysis process was carried out with double transducer hand piece which sends out focused ultrasonic waves to the adipose tissue beneath the skin. This section lasted for 30 to 45 minutes with frequencies of $20 \mathrm{~Hz}$ to $60 \mathrm{~Hz}$ depending on the adipose tissue caliper, and at powers of 0.5 to 3 $\mathrm{w} / \mathrm{cm}^{2}$ depending on the patient's temperature tolerance. Afterward, a 6-minute vacuum drainage was done with a vacuum hand piece that moved centrifugally from the umbilicus to the epigastric and hypogastric regions. This normally promotes evacuation of fat particles in the way of abdominal lymphatic drainage to epigastric and inguinal lymph nodes. To help better extraction of fat droplets, an additional vacuum drainage for 15 to 30 minutes was performed three days after each lipolysis sessions. Patients were advised to limit consumption of simple carbohydrate foods in three days post-lipolysis.

\section{Circumference Measurements}

The change in abdominal circumference was indicated to evaluate the efficacy of treatment. The largest abdominal girth and 2 lines at $4 \mathrm{~cm}$ to $7 \mathrm{~cm}$ distance above and under it were located as fixed points of measurements. The mean value of the 3 fixed lines was considered the abdominal circumference. Measurements were taken at baseline, before each lipolysis session and 1 week as well as 3 months after the final session of treatment.

At each lipolysis session, the difference between pretreatment to posttreatment abdominal circumferences was defined as "per-session circumference reduction." Moreover, "total circumference reduction" was assessed by comparing circumference values at baseline to oneweek post final treatment. At 3-month follow-up visit, abdominal circumference was measured once more and compared to the baseline value to identify "final circumference reduction." Treatment discomfort and side effects such as surface irregularities and blister formation were assessed in each session of treatment as well as follow-up visit. Patients were asked to determine their treatment satisfaction at one-week post final treatment and follow-up visits based on an arbitrary scale: high satisfaction, partial satisfaction, no change and dissatisfaction.

\section{Statistical Analysis}

Statistical analysis was carried out using SPSS version 16 (SPSS Inc., Chicago, IL). The mean per-session circumference reductions were compared using paired $t$ test. The Pearson correlation coefficient was used to assess the effects of the patient's age, baseline abdominal circumference and body mass index (BMI) on circumference reduction. Differences were considered statistically significant when $P<.05$.

\section{Results}

A total of 194 external ultrasonic lipolysis procedures were performed on 28 subjects. Each patient underwent 3 to 8 treatment sessions (median: $8, \mathrm{Q}_{25}: 6, \mathrm{Q}_{75}: 8$ ). Comparison of pretreatment and posttreatment weights indicates no significant difference $(P>.05)$, although an increase in the mean value of weight was observed in the follow-up visit (71.6 kg vs $88.0 \mathrm{~kg}, P>.05$ ).

An average decrease of circumference value of $1.89 \mathrm{~cm}$ (95\% CI: $1.63-2.02 \mathrm{~cm})$ was estimated for each session of treatment, that was statistically significant $(P<.001)$. However, according to Table 1 which illustrates the mean

Table 1. Abdominal Circumference Changes Based on Ultrasonic Lipolysis Treatments

\begin{tabular}{|c|c|c|c|c|}
\hline \multirow[t]{2}{*}{ Treatment Session } & \multirow[t]{2}{*}{ Number } & \multicolumn{2}{|c|}{$\begin{array}{c}\text { Abdominal Circumference }(\mathrm{cm}) \\
\text { Mean } \pm \text { SD or SE }\end{array}$} & \multirow{2}{*}{$\begin{array}{l}\text { Per-session Circumference } \\
\text { Reduction }(\mathrm{cm})\end{array}$} \\
\hline & & Pretreatment & Posttreatment & \\
\hline First & 28 & $100.5 \pm 9.5$ & $97.9 \pm 9.7$ & 1.29 \\
\hline Second & 28 & $98.8 \pm 9.5$ & $97.1 \pm 9.2$ & 0.88 \\
\hline Third & 28 & $97.4 \pm 9.3$ & $95.7 \pm 9.2$ & 0.86 \\
\hline Fourth & 26 & $96.7 \pm 8.6$ & $95.2 \pm 8.6$ & 0.70 \\
\hline Fifth & 25 & $96.5 \pm 8.5$ & $95.2 \pm 8.3$ & 0.57 \\
\hline Sixth & 24 & $95.4 \pm 8.3$ & $93.4 \pm 8.1$ & 0.86 \\
\hline Seventh & 20 & $94.4 \pm 8.1$ & $92.1 \pm 8.2$ & 0.86 \\
\hline Eighth & 16 & $91.9 \pm 7.9$ & $90.5 \pm 7.3$ & 0.40 \\
\hline
\end{tabular}




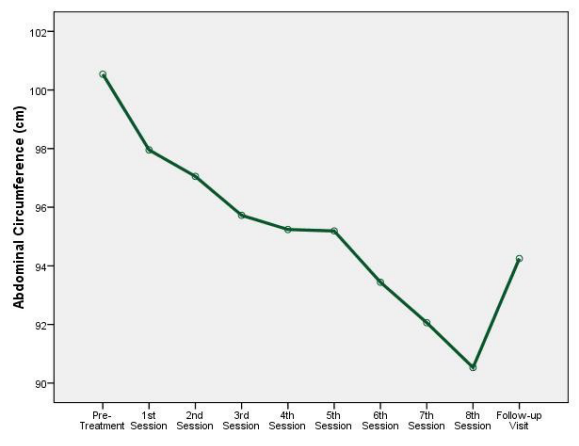

Figure 1. Trend of Mean Abdominal Circumference Values During Treatments and Follow-up.

of per-session circumference reduction in each treatment, no statistical difference was seen between different sessions of ultrasonic lipolysis $(P>.05)$. Furthermore, there was no significant correlation between per-session circumference reduction and the patient's age $(r=0.015$, $P=.954)$, baseline abdominal circumference $(r=0.350$, $P=.068)$ as well as BMI $(\mathrm{r}=0.378, P=.134)$.

The mean value of total circumference reduction was 8.21 $\mathrm{cm}(95 \% \mathrm{CI}: 6.38-10.04 \mathrm{~cm})$ that was significantly different from baseline value $(P<.001)$. Although abdominal circumference reduction was partially reversed in 18 cases (64.3\%), the mean value of final circumference reduction in comparison to the baseline measure was $7 \mathrm{~cm}(95 \% \mathrm{CI}$ : 3.2-10.8) which was again statistically significant $(P<.001$; Figure 1).

There were no significant adverse effects such as burning or blister formation and the procedure was well tolerated. None of the patients complained of pain and there were no request for discontinuation of lipolysis procedure. Finally, at the end of the lipolysis sessions, 16 subjects (76.2\%) were fully satisfied with the result which finally declined to 9 subjects $(42.9 \%)$ at the 3 -month follow-up visit.

\section{Discussion}

Despite an increased demand for noninvasive ultrasonic body contouring, there is a lack of evidence about its final clinical consequences. To our knowledge, limited trials have been performed to evaluate cellulite reduction by noninvasive ultrasonic lipolysis and most of the available studies are company supported. This study has shown that external ultrasonic lipolysis can effectively reduce abdominal cellulite deposition. We conclude that an average of $1.8 \mathrm{~cm}$ decrease in circumference girth can be expected during a session of ultrasonic lipolysis. Surprisingly, receiving more episodes of lipolysis did not result in a greater circumference reduction. For example, the mean circumference reductions of the second and the seventh lipolysis sessions were $0.88 \mathrm{~cm}$ and $0.86 \mathrm{~cm}$, respectively. Previous studies over efficacy of external ultrasonic lipolysis have been controversial. In a study by Teitelbaum et $\mathrm{al}^{12}$ on 164 patients, an average reduction of $2 \mathrm{~cm}$ per treatment in circumference girth is reported. Similar result was observed by Moreno-Morago et $\mathrm{al}^{10}$ that demon- strated $1.8 \mathrm{~cm}$ circumference reduction per session with steady response during subsequent treatments. However, the result of a trial in Asians was disappointing as Shek et $\mathrm{al}^{11}$ demonstrated an increase of $2.03 \mathrm{~cm}$ in the abdominal circumference after three sessions of ultrasonic lipolysis. Diversity of body size as well as measurement bias due to laxity of abdominal skin after lipolysis is suggested to explain this difference.

Owing to tight control of the weight, we eliminated the bias effects of weight loss on circumference reduction. Although a prior report has proposed to select patients with normal to overweight BMIs $(\leq 30),{ }^{9}$ marginal correlation was found between the circumference reduction and the BMI. Likewise, the baseline abdominal girth which can be a representative of subcutaneous fat caliper, has limited effect on the final result. Therefore, our data supported the efficacy of focused ultrasonic lipolysis on obese patients with BMI over 30 .

Three months after the last treatment, we found substantial persistent effect of ultrasonic lipolysis with maintenance of $7 \mathrm{~cm}$ mean circumference reduction, albeit insignificant amount of regression was developed in $64 \%$ of patients. Persistence of $3.95 \mathrm{~cm}$ cumulative circumference reduction has also been shown by Moreno-Morago et al ${ }^{10}$ after 1-month follow up of the patients. ${ }^{10}$ However, in the study by shek et $\mathrm{al},{ }^{11}$ the average abdominal circumference at baseline and at 3-month follow-up visit was 96.6 $\mathrm{cm}$ and $96.2 \mathrm{~cm}$, respectively which can be the consequence of overall poor results. Further trials to evaluate the long-lasting outcomes of focused ultrasonic lipolysis can be helpful.

In this open-label trial, we encountered some limitations such as lack of randomized control group, ultrasonographic subcutaneous fat caliper measurement, and hepatic evaluation of fat deposition in addition to serum lipid profile assessment.

In conclusion, we confirmed the efficacy of focused ultrasonic lipolysis in the treatment of abdominal cellulite with persistent effect. Regarding the minimal side effects of focused ultrasound lipolysis and also no need for recovery time, this procedure can be used as an alternative or in combination with other invasive or noninvasive body contouring procedures.

\section{Acknowledgments}

This project was supported by a grant from Shahid Beheshti University of Medical Science. The authors express sincere appreciation to the laser application in Medical Sciences Research Center of Shahid Behesti University of Medical Science and would like to especially thank Alireza Gholibeikian for his editorial support and Farzaneh Akbari for her technical support.

\section{Conflict of Interest}

The authors have no conflict of interest to declare.

\section{Funding Source}

Laser application in Medical Sciences Research Center, 
Shohada-e Tajrish hospital, Shahid Beheshti University of Medical Science, Tehran.

\section{References}

1. Khan MH, Victor F, Rao B, Sadick NS. Treatment of cellulite: Part II. Advances and controversies. J Am Acad Dermatol. 2010;62(3):373-384.

2. Wang HD, Zheng JH, Deng CL, Liu QY, Yang SL. Fat embolism syndromes following liposuction. Aesthetic Plast Surg. 2008;32(5):731-736. doi:10.1007/s00266008-9183-1.

3. Terranova C, Sartore D, Snenghi R. Death after liposuction: case report and review of the literature. Med Sci Law. 2010;50(3):161-163. doi:10.1258/ msl.2010.100010.

4. Rao RB, Ely SF, Hoffman RS. Deaths related to liposuction. N Engl J Med. 1999;340:1471-1475. doi:10.1056/nejm199905133401904.

5. Paul M, Mulholland RS. A new approach for adipose tissue treatment and body contouring using radiofrequency-assisted liposuction. Aesthetic Plast Surg. 2009;33(5):687-694. doi:10.1007/s00266-0099342-z.

6. Brown SA, Greenbaum L, ShtukmasterS, ZadokY,BenEzra S, Kushkuley L. Characterization of nonthermal focused ultrasound for noninvasive selective fat cell disruption (lysis): technical and preclinical assessment. Plast Reconstr Surg. 2009;124(1):92-101. doi:10.1097/prs.0b013e31819c59c7.

7. Coleman KM, Coleman WP 3rd, Benchetrit A. Non-invasive, external ultrasonic lipolysis. Semin Cutan Med Surg. 2009;28(4):263-267. doi: 10.1016/j. sder.2009.10.004.

8. Fatemi A. High-intensity focused ultrasound effectively reduces adipose tissue. Semin Cutan Med Surg. 2009;28(4):257-62.

9. Hotta TA. Nonsurgical body contouring with focused ultrasound. Plast Surg Nurs. 2010;30(2):77-82. doi:10.1097/psn.0b013e3181dee9c9.

10. Moreno-Moraga J, Valero-Altés T, Riquelme AM, Isarria-Marcosy MI, de la Torre JR. Body contouring by non-invasive transdermal focused ultrasound. Lasers Surg Med. 2007;39(4):315-323. doi:10.1002/ $1 \mathrm{sm} .20478$

11. Shek S, Yu C, Yeung CK, Kono T, Chan HH. The use of focused ultrasound for non-invasive body contouring in Asians. Lasers Surg Med. 2009;41(10):751-759.

12. Teitelbaum SA, Burns JL, Kubota J, et al. Noninvasive body contouring by focused ultrasound: safety and efficacy of the Contour I device in a multicenter, controlled, clinical study. Plast Reconstr Surg. 2007;120(3):779-789. 